\title{
Canards Solutions of Difference Equations with Small Step Size
}

\author{
Abir EL-RABIH*i‡
}

June 2002

Abstract: We consider difference equations of the form

$$
y(\varepsilon, x+\varepsilon)=x y(\varepsilon, x)+\varepsilon f(\varepsilon, x, a(\varepsilon), y(\varepsilon, x)),
$$

where $\varepsilon>0$ and small, $a(\varepsilon)$ is a scalar function of $\varepsilon$ to be determined and $f$ is an analytic function of $\varepsilon, x, a$ and $y$, also near $\varepsilon=0$. We show the existence of values of $a(\varepsilon)$ for which the difference equation admits canard solutions $y(\varepsilon, x)$, i.e. solutions on certain domains containing $x=1$ that are bounded uniformly with respect to $\varepsilon$. We study the asymptotic behavior of the canards as $\varepsilon \rightarrow 0$.

We also give an example of linear difference equations of the above form where $f$ is replaced by $a(\varepsilon)+g(x), g$ some piecewise continuous function on some interval containing 1. Here we study solutions on discrete sets and compare their properties with the analytic case.

The main tool of the proof is the construction, on suitable spaces of analytic functions, of a right inverse of the operator $\mathcal{L}_{\varepsilon}$ given by

$$
\mathcal{L}_{\varepsilon}(a, y)(\varepsilon, x)=\frac{1}{\varepsilon}[y(\varepsilon, x+\varepsilon)-(x+\varepsilon A(x)) y(\varepsilon, x)]-B(x) a(\varepsilon),
$$

where $B(1) \neq 0$.

Keywords: Difference equation; Difference operator; Inverse operator; Canard solution; Sum of a function

AMS Classification: 39A, 47B39

Acknowledgement: The author would like to thank her advisor Reinhard Schäfke for his continuous support. She is also grateful to Augustin Fruchard for his remarks. The author is especially indebted to the National Council of Scientific Research in Lebanon for financially supporting her Ph.D work as a whole and this research in particular.

${ }^{*}$ UFR de Mathématique et d'Informatique, Université Louis Pasteur, 7 rue René Descartes, 67084 Strasbourg cedex, France.

${ }^{\dagger}$ Tel:+(33)03.90.24.02.68 Fax:+(33)03.90.24.03.28 E-mail:elrabih@math.u-strasbg.fr

$\ddagger$ Scholarship holder of the National Council of Scientific Research in Lebanon 


\section{Introduction}

We consider a (family of) difference equations of the form

$$
y(\varepsilon, x+\varepsilon)=x y(\varepsilon, x)+\varepsilon f(\varepsilon, x, a(\varepsilon), y(\varepsilon, x)),
$$

where

- $\left.\varepsilon \in] 0, \varepsilon_{0}\right], \varepsilon_{0}$ being some fixed small positive number,

- $a$ is some scalar function of $\varepsilon$ to be determined,

- $y$ is some complex function of $\varepsilon$ and the complex variable $x$ and

- $f$ is an analytic function of $\varepsilon$ in a neighborhood of $\left[0, \varepsilon_{0}\right]$, and of the variables $a, x, y$.

Equation (1) is closely related to the static bifurcation problem [5]

$$
y_{n+1}=x y_{n}+\varepsilon f\left(\varepsilon, x, a(\varepsilon), y_{n}\right),
$$

where $y_{0}$ is arbitrary, $x$ is a real or complex parameter as well as the variable $y$ and $f$ is some $C^{\infty}$ or analytic function. For $x \neq 1$ and sufficiently small $\varepsilon,(2)$ admits, by the implicit function theorem, a unique fixed point $y^{*}$ close to 0 . This fixed point is stable and attractive if $0<x<1$, but unstable and repulsive if $x>1$ ( $\operatorname{cf}[10]$, p. 22). If $x=1$, a fixed point might exist for small $\varepsilon$ and suitably chosen $a(\varepsilon)$, but its stability properties depend upon properties of $f$. In any case, $x=1$ is a parameter value associated to a stability loss of the fixed point and thus bifurcation might occur; this is the so called static bifurcation because $x$ remains fixed.

The study of equation (1) is equivalent to a certain dynamic bifurcation problem [9], if considered on the discrete set of all $x=x_{0}+n \varepsilon$, where $x_{0}$ is an arbitrarily fixed point. Putting $x_{n}=x_{0}+n \varepsilon, y_{n}=y\left(x_{n}\right)$, (1) can be written as:

$$
x_{n+1}=x_{n}+\varepsilon, y_{n+1}=x_{n} y_{n}+\varepsilon f\left(\varepsilon, x_{n}, a(\varepsilon), y_{n}\right) .
$$

Here, the parameter value $x$ changes slowly with $n$; the question is how closely this dynamic bifurcation problem is related to the static one. We refer to [9] for a more detailed discussion of static and dynamic bifurcation.

In the present article, we are interested in finding "canard" [1], [2], [3], [5], [7] values and solutions of (1), i.e. values $a(\varepsilon)$ for which (1) admits well behaved (see below) solutions that are defined on sets of $x$-values containing points $x>1$ and $x<1$. These canards can be studied for discrete subsets $x_{0}, \ldots, x_{N}$ of the real axis, $x_{0}<1<x_{N}$ or for complex domains containing $x=1$. The existence of a canard value and solution for a domain containing $x=1$ is much more restrictive than the existence for just discrete subsets; it requires that the canard value can be chosen the same for all discrete subsets of the domain.

More precisely, our aim is to show the existence of functions $a(\varepsilon)$ for which $(1)$ admits analytic solutions $y$ bounded for small $\varepsilon>0$ and on certain bounded $x$-domains containing the "critical" point $x=1$.

In the discrete (real) case, we just consider a linear form of (1). We prove the existence of canards in this case and show that the interval of possible canard values has exponentially small length for a given discrete set $x_{0}, \ldots, x_{N}$. In an example, we show that for non analytic $f$, the canard values cannot be chosen independent of the discrete set. We also study the asymptotic behavior as $\varepsilon \rightarrow 0$ of the family $y_{\varepsilon}$ of solutions of (1). 
Observe that we chose to discuss the specific family of equations (1) to simplify matters but our results could be easily generalized to families of difference equations of the form

$$
y(\varepsilon, x+\varepsilon)=g(x) y(\varepsilon, x)+\varepsilon f(\varepsilon, x, a(\varepsilon), y(\varepsilon, x)),
$$

having the same properties as (1) and where $g(x)$ is some analytic function having a simple zero.

Some recent work on solutions for difference equations and invariant curves can be found in [6], [8], [9]. However, it seems that that no work has been yet done on "ephemeral canard" solutions that are discussed in this article. By "ephemeral canard" solutions, we mean "canard" solutions that are short-lived in the sense that the $a$-interval of existence has only exponentially small length.

The principal result of this work is the existence of analytic solutions $a(\varepsilon), y(\varepsilon, x)$ bounded on certain $x$-domains to be discussed later. The proof uses some right inverse of a certain difference operator and the fixed point theorem.

In order to transform (1) into a fixed point equation, we first need to construct, on suitable spaces of analytic functions, a right inverse of the operator $\mathcal{L}_{\varepsilon}$ given by

$$
\mathcal{L}_{\varepsilon}(a, y)(\varepsilon, x)=\frac{1}{\varepsilon}[y(\varepsilon, x+\varepsilon)-(x+\varepsilon A(x)) y(\varepsilon, x)]-B(x) a(\varepsilon),
$$

where $A(x)$ and $B(x)$ are holomorphic functions. Here we need the assumption that $B(1) \neq 0$. The construction of this inverse operator is done at the end of section three after several preparatory steps.

First, we construct a right inverse $T_{\varepsilon}$ of the operator $\tilde{\mathcal{L}}_{\varepsilon}$ given by

$$
\tilde{\mathcal{L}}_{\varepsilon} y(\varepsilon, x)=\frac{1}{\varepsilon}[y(\varepsilon, x+\varepsilon)-(x+\varepsilon A(x)) y(\varepsilon, x)] .
$$

Next, we discuss $x$-domains for which $T_{\varepsilon}$ defines a bounded operator. We introduce inverse operators $T_{\varepsilon}^{ \pm}$of $\tilde{\mathcal{L}}_{\varepsilon}$ that are bounded on certain $x$-domains, on the left respectively the right of $x=1$, to be described later. Then, we show that, given $g(x, \varepsilon)$, the functions $T_{\varepsilon}^{+} h$ and $T_{\varepsilon}^{-} h$ are the same for $h(x, \varepsilon)=B(x) a(\varepsilon)+g(\varepsilon, x)$ provided $a(\varepsilon)$ has a certain value; here we need that $B(1) \neq 1$. This leads to an explicit formula for a right inverse of $\mathcal{L}_{\varepsilon}$.

In section four, we use the newly constructed right inverse of $\tilde{\mathcal{L}}_{\varepsilon}$ and the fixed point principle to prove the existence of bounded analytic solutions of equation (1) in the general (non linear) case.

\section{The Problem on The Real Line}

\subsection{The Real Linear Case}

We consider the boundary value problem

$$
y(x+\varepsilon)=x y(x)+a(\varepsilon)+\varepsilon f(\varepsilon, x), y(\alpha)=y_{\alpha}(\varepsilon), y(\beta)=y_{\beta}(\varepsilon),
$$

where $y_{\alpha}(\varepsilon)$ and $y_{\beta}(\varepsilon)$ are bounded and $\left.1 \in\right] \alpha, \beta[$. We also assume that $f$ is bounded on $[\alpha, \beta]$. We call a discrete solution $(a(\varepsilon), y(\varepsilon, x))$ of $(7)$ on $[\alpha, \beta]$ a value $a(\varepsilon)$ and a function $y(\varepsilon, x)$ defined on the finite set $\{\alpha, \alpha+\varepsilon, \ldots, \beta-\varepsilon, \beta\}$. Observe that such a discrete solution is only defined if $\beta-\alpha$ is a multiple of $\varepsilon$. Moreover, we say that it is bounded if both $a(\varepsilon)$ and $y(\varepsilon, x)$ are bounded on the set of all $\varepsilon, x$ such that $(\beta-\alpha) / \varepsilon$ respectively $(x-\alpha) / \varepsilon$ are integers. 
Theorem 1 There exits a discrete bounded solution $(a(\varepsilon), y(\varepsilon, x))$ of $(7)$ on $[\alpha, \beta]$.

Proof. We set $x_{0}=\alpha, \varepsilon=(\beta-\alpha) / n_{1}, n_{1} \in \mathbb{N}$ and $x_{n}=\alpha+n \varepsilon, n=0, \ldots, n_{1}$. Then (7) admits a discrete solution given by

$$
y\left(\varepsilon, x_{n}\right)=\sum_{k=0}^{n-1}\left[\left(a(\varepsilon)+\varepsilon f\left(\varepsilon, x_{k}\right)\right) \prod_{m=k+1}^{n-1} x_{m}\right]+y_{\alpha}(\varepsilon) \prod_{m=0}^{n-1} x_{m}, n=1, \ldots, n_{1},
$$

where $a(\varepsilon)$ is determined by $y(\beta)=y_{\beta}(\varepsilon)$, i.e.

$$
a(\varepsilon)=\frac{y_{\beta}(\varepsilon)-y_{\alpha}(\varepsilon) \prod_{m=0}^{n_{1}-1} x_{m}-\varepsilon \sum_{k=0}^{n_{1}-1} f\left(\varepsilon, x_{k}\right) \prod_{m=k+1}^{n_{1}-1} x_{m}}{\sum_{k=0}^{n_{1}-1} \prod_{m=k+1}^{n_{1}-1} x_{m}},
$$

Obviously,

$$
\begin{gathered}
|a(\varepsilon)| \leq \varepsilon \max _{[\alpha, \beta]}|f(\varepsilon, x)|+\frac{\left|y_{\beta}(\varepsilon)-y_{\alpha}(\varepsilon) \prod_{m=0}^{n_{1}-1} x_{m}\right|}{\left|\sum_{k=0}^{n_{1}-1} \prod_{m=k+1}^{n_{1}-1} x_{m}\right|}, \text { and } \\
\left|y\left(\varepsilon, x_{n}\right)\right| \leq\left(|a(\varepsilon)|+\varepsilon \max _{[\alpha, \beta]}|f|\right) \sum_{k=0}^{n-1} \prod_{m=k+1}^{n-1} x_{m}+\left|y_{\alpha}(\varepsilon) \prod_{m=0}^{n-1} x_{m}\right| .
\end{gathered}
$$

In order to show that the corresponding solution $(a(\varepsilon), y(\varepsilon, x))$ is bounded, we let

$$
g\left(x_{k}\right)=\prod_{m=k+1}^{n-1} x_{m} ; k=0, \ldots, n-1
$$

then $g\left(x_{k-1}\right)=x_{k} g\left(x_{k}\right)$ and $g\left(x_{n-1}\right)=1$. It is natural to look for an analytic solution of the homogeneous equation $g(x-\varepsilon)=x g(x)$. We introduce a new function $z(\varepsilon, x)$ given by

$$
z(\varepsilon, x)=z\left(\varepsilon, x_{n-1}\right)+\varepsilon \log g(x) .
$$

Then, $z$ satisfies $z(\varepsilon, x-\varepsilon)=z(\varepsilon, x)+\varepsilon \log x$. That is, $z$ is a so called "sum" of the complex function $-\log x$. It is known that such a sum exits and is close to an antiderivative of $-\log x$. Indeed, its existence follows from a theorem of A.Fruchard [4]. Moreover, it is unique except for an $\varepsilon$-periodic function. It is also known that any sum tending to 0 as $x \rightarrow 0^{+}$is of the form $x-x \log x+\mathcal{O}(\varepsilon)$. For example, the function $z(\varepsilon, x)$, given by

$$
z(\varepsilon, x)=-\left(x+\frac{1}{2} \varepsilon\right) \log \varepsilon-\varepsilon \log \Gamma\left(\frac{x}{\varepsilon}+1\right)
$$

has the properties $z(\varepsilon, x-\varepsilon)=z(\varepsilon, x)+\varepsilon \log x$ and $z(\varepsilon, x)=x-x \log x+\mathcal{O}(\varepsilon)$ as $\varepsilon \rightarrow 0^{+}$ uniformly for $x$ on bounded sets. Then, it is a sum of $-\log x$. For the moment, we just need

$$
z(x)=x-x \log x+\mathcal{O}(\varepsilon)
$$

Then

$$
\begin{aligned}
& \prod_{m=1}^{n_{1}-1} x_{m}=\exp \left(\frac{1}{\varepsilon}\left(z\left(\varepsilon, x_{0}\right)-z\left(\varepsilon, x_{n_{1}-1}\right)\right)\right)=\exp \left(\frac{1}{\varepsilon}(\tilde{z}(\alpha)-\tilde{z}(\beta))+\mathcal{O}(1)\right), \text { and } \\
J= & \sum_{k=0}^{n_{1}-1} \prod_{m=k+1}^{n_{1}-1} x_{m}=\sum_{k=0}^{n_{1}-1} \exp \left(\frac{1}{\varepsilon}\left(z\left(\varepsilon, x_{k}\right)-z\left(\varepsilon, x_{n_{1}-1}\right)\right)\right)=\sum_{k=0}^{n_{1}-1} \exp \left(\frac{1}{\varepsilon}\left(\tilde{z}\left(x_{k}\right)-\tilde{z}(\beta)\right)+\mathcal{O}(1)\right),
\end{aligned}
$$


where $\tilde{z}(x)=x-x \log x$. As $\tilde{z}$ is increasing on $[\alpha, 1]$,

$$
J=\frac{1}{\varepsilon} \int_{\alpha}^{\beta} \exp \left(\frac{1}{\varepsilon}(\tilde{z}(t)-\tilde{z}(\beta))+\mathcal{O}(1)\right) d t
$$

which, using Laplace's method, is shown to be $\mathcal{O}\left(\varepsilon^{-1 / 2} \exp \left(\frac{1}{\varepsilon}(1-\tilde{z}(\beta))\right)\right)$.

Since also $y_{\alpha}$ and $y_{\beta}$ are bounded, we have

$$
\begin{gathered}
y_{\beta} / J=\mathcal{O}\left(\varepsilon^{1 / 2} \exp \left(\frac{1}{\varepsilon}(\tilde{z}(\beta)-1)\right)\right), \text { and } \\
y_{\alpha} \prod_{m=0}^{n_{1}-1} x_{m} / J=\mathcal{O}\left(\varepsilon^{1 / 2} \exp \left(\frac{1}{\varepsilon}(\min (\tilde{z}(\alpha), \tilde{z}(\beta))-1)\right)\right) .
\end{gathered}
$$

This implies that $|a(\varepsilon)| \leq \varepsilon \max _{[\alpha, \beta]}|f(\varepsilon, x)|+g(\varepsilon, x)$, where $g(\varepsilon, x)$ is an exponentially small function.

Then $a$ is bounded since $f$ is bounded on $[\alpha, \beta]$. Next we show that $y$ is also bounded. For $x_{n} \leq 1$, we have

$$
\left|y\left(\varepsilon, x_{n}\right)\right| \leq 2 \varepsilon \max _{[\alpha, \beta]}|f| \sum_{k=0}^{n-1} \prod_{m=k+1}^{n-1} x_{m}+g \sum_{k=0}^{n-1} \prod_{m=k+1}^{n-1} x_{m}+\left|y_{\alpha}(\varepsilon)\right| \prod_{m=0}^{n-1} x_{m} .
$$

Letting $J_{n}=\sum_{k=0}^{n-1} \prod_{m=k+1}^{n-1} x_{m}$, we have as before

$$
J_{n}=\frac{1}{\varepsilon} \int_{\alpha}^{x} \exp \left(\frac{1}{\varepsilon}(\tilde{z}(t)-\tilde{z}(x-\varepsilon))+\mathcal{O}(1)\right) d t
$$

which is of the order of $\frac{1}{\varepsilon}$. The product

$$
\prod_{m=1}^{n-1} x_{m}=\exp \left(\frac{1}{\varepsilon}(\tilde{z}(\alpha)-\tilde{z}(x-\varepsilon))+\mathcal{O}(1)\right)=\mathcal{O}(1)
$$

since $\tilde{z}$ is increasing on $[\alpha, 1]$. This shows that $y$ is bounded on $[\alpha, 1]$.

The proof that $y$ is bounded on $[1, \beta]$ is symmetric to the above case. We rewrite (7) as

$$
y(x)=\frac{1}{x} y(x+\varepsilon)-\frac{1}{x}(a(\varepsilon)+\varepsilon f(\varepsilon, x))
$$

We keep $\varepsilon$ and $n_{1}$ as in the above case for $x_{n} \leq 1$. However, we set $x_{0}=\beta$ and $x_{n}=$ $\beta-n \varepsilon, n=0, \ldots, n_{1}$. Then (7) admits a discrete solution given by

$$
y\left(\varepsilon, x_{n}\right)=\sum_{k=0}^{n-1}\left[\frac{-1}{x_{k}}\left(a(\varepsilon)+\varepsilon f\left(\varepsilon, x_{k}\right)\right) \prod_{m=k+1}^{n-1} \frac{1}{x_{m}}\right]+y_{\beta}(\varepsilon) \prod_{m=0}^{n-1} \frac{1}{x_{m}}, n=1, \ldots, n_{1} .
$$

Then

$$
\left|y\left(\varepsilon, x_{n}\right)\right| \leq C_{1} \sum_{k=0}^{n-1} \prod_{m=k+1}^{n-1} \frac{1}{x_{m}}+C_{2} \prod_{m=1}^{n-1} \frac{1}{x_{m}},
$$

where $C_{1}$ and $C_{2}$ are constants.

By a similar argument to the case $x \in[\alpha, 1]$, we have

$$
\prod_{m=1}^{n-1} \frac{1}{x_{m}}=\exp \left(\frac{1}{\varepsilon}(\tilde{z}(\beta)-\tilde{z}(x-\varepsilon))+\mathcal{O}(1)\right)
$$




$$
\sum_{k=0}^{n-1} \prod_{m=k+1}^{n-1} \frac{1}{x_{m}}=\frac{1}{\varepsilon} \int_{\beta}^{x} \exp \left(\frac{1}{\varepsilon}(\tilde{z}(t)-\tilde{z}(x-\varepsilon))+\mathcal{O}(1)\right) d t
$$

where the function $\tilde{z}$ is monotonically decreasing on $[1, \beta]$. This shows that $y$ is bounded on $[1, \beta]$.

Theorem 2 If $\left(a_{1}(\varepsilon), y_{1}(\varepsilon, x)\right)$ and $\left(a_{2}(\varepsilon), y_{2}(\varepsilon, x)\right)$ are two bounded discrete solutions of (7), then $a_{1}-a_{2}$ is exponentially small.

Proof. Let $d=y_{1}-y_{2}$. Then $d$ satisfies the boundary value problem

$d(x+\varepsilon)=x d(x)+a_{1}(\varepsilon)-a_{2}(\varepsilon), d(\alpha)=d_{\alpha}(\varepsilon)=y_{1}(\varepsilon, \alpha)-y_{2}(\varepsilon, \alpha), d(\beta)=d_{\beta}(\varepsilon)=y_{1}(\varepsilon, \beta)-y_{2}(\varepsilon, \beta)$

Since $d_{\alpha}(\varepsilon)$ and $d_{\beta}(\varepsilon)$ are bounded, we have by (8) and (9)

$$
\begin{gathered}
d\left(\varepsilon, x_{n}\right)=\left(a_{1}(\varepsilon)-a_{2}(\varepsilon)\right)\left(\sum_{k=0}^{n-1} \prod_{m=k+1}^{n-1} x_{m}\right)+d_{\alpha}(\varepsilon) \prod_{m=0}^{n-1} x_{m}, n=1, \ldots, n_{1}, \\
a_{1}(\varepsilon)-a_{2}(\varepsilon)=\frac{d_{\beta}(\varepsilon)-d_{\alpha}(\varepsilon) \prod_{m=0}^{n_{1}-1} x_{m}}{\sum_{k=0}^{n_{1}-1} \prod_{m=k+1}^{n_{1}-1} x_{m}}
\end{gathered}
$$

respectively.

By (10) and (11), the quotients $1 / \sum_{k=0}^{n_{1}-1} \prod_{m=k+1}^{n_{1}-1} x_{m}$ and $\prod_{m=1}^{n_{1}-1} x_{m} / \sum_{k=0}^{n_{1}-1} \prod_{m=k+1}^{n_{1}-1} x_{m}$ are exponentially small. This finishes the proof.

Next, we would like to see what happens if we change the discrete set of definition of our solution by shifting it to the right, say to $\{\alpha+\theta \varepsilon, \alpha+(\theta+1) \varepsilon, \ldots, \beta+(\theta-1) \varepsilon, \beta+\theta \varepsilon\}$. We have done some numerical experiments that illustrates the behavior of $a$. We develop this in the below example.

\subsection{Example}

We consider the boundary value problem

$$
y(x+\varepsilon)=x y(x)+a(\varepsilon)+\varepsilon \operatorname{sign}(x-1), y(\alpha)=y(\beta)=0,
$$

where $\operatorname{sign}(x-1)=1$ if $x \geq 1,-1$ if $x<1$ and $0<\alpha<1<\beta$. We know that there exits a unique value of $a(\varepsilon)$ for which this boundary value problem admits a family of discrete solutions $y_{\varepsilon}(x)_{\varepsilon \in\left[0, \varepsilon_{0}\right]}$ bounded on $[\alpha, \beta]$ and that tends to the slow curve $y_{0}=0$ as $\varepsilon \rightarrow 0$. However, we are interested in the behavior of $a$ as we move a little our boundary conditions. In other words, what will become the new value of $a$ if we consider the new boundary conditions $y(\alpha+\theta \varepsilon)=y(\beta+\theta \varepsilon)=0$, where $\theta \in[-k, k]$ and $\alpha+\theta \varepsilon<1$ ?

Numerical experiments have shown that $a=a(\varepsilon, \theta)$ depends on $\theta$ in a "quasi-periodic" way, i.e. $a(\varepsilon, \theta+1)-a(\varepsilon, \theta)$ is exponentially small (See figure 1 on page 7 and figure 2 on page 8).

Indeed, this "quasi-periodicity" is easily inferred from the above section. To see this, we denote $\left(a_{\theta}(\varepsilon), y_{\theta}(\varepsilon, x)\right)$ the discrete solution of the boundary value problem

$$
y(x+\varepsilon)=x y(x)+a(\varepsilon)+\varepsilon \operatorname{sign}(x-1), y(\alpha+\theta \varepsilon)=y(\beta+\theta \varepsilon)=0,
$$


and $\left(a_{\theta+1}(\varepsilon), y_{\theta+1}(\varepsilon, x)\right)$ the discrete solution of the boundary value problem

$$
y(x+\varepsilon)=x y(x)+a(\varepsilon)+\varepsilon \operatorname{sign}(x-1), y(\alpha+(\theta+1) \varepsilon)=y(\beta+(\theta+1) \varepsilon)=0 .
$$

Then both solutions can be extended to discrete solutions of the boundary value problem on the interval $[\alpha+\theta \varepsilon, \beta+(\theta+1) \varepsilon]$.

$y(x+\varepsilon)=x y(x)+a(\varepsilon)+\varepsilon \operatorname{sign}(x-1), y(\alpha+\theta \varepsilon)=y_{\alpha, \theta}<\infty, y(\beta+(\theta+1) \varepsilon)=y_{\beta, \theta+1}<\infty$.

Then our claim is shown by theorem 2 .

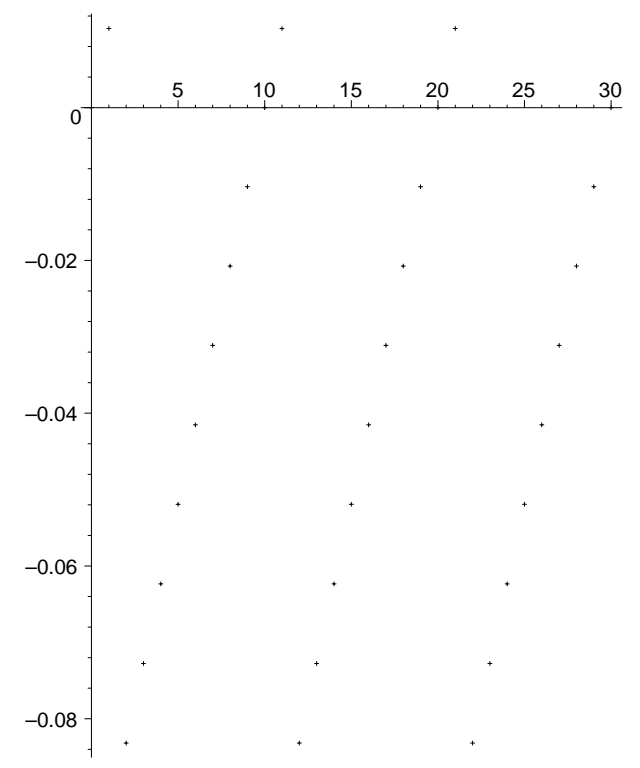

Figure 1: The function $a_{i}-a_{0}$

In the following sections, we will construct analytic solutions of equation (1) defined on certain bounded $x$-domains; these contain, of course, real intervals $[\alpha, \beta]$ of the form studied above. As a consequence, the function $a(\varepsilon, \theta)$ is not only quasi-periodic in this case, but - as a consequence of theorem 2 - exponentially close to some constant (i.e. independent of $\theta$ ). This was one of the motivations to study the existence of such solutions defined on domains.

\section{Construction of an Inverse of $\left(\mathcal{L}_{\varepsilon}\right)$}

We first need to construct a right inverse of $\mathcal{L}_{\varepsilon}$. In other words, given $\varepsilon>0$ and some domain $D \subset \mathbb{C}$, that we describe later, we want to construct a bounded linear operator $\gamma_{\varepsilon}$, on the banach space $\bar{H}(D)$, of the holomorphic bounded funtions on $\mathrm{D}$, with the following property: Given $h \in \bar{H}(D)$, then $\gamma_{\varepsilon} h$ satisfies $\mathcal{L}_{\varepsilon} \gamma_{\varepsilon} h=h$.

We consider the equation

$$
\tilde{\mathcal{L}}_{\varepsilon} y(\varepsilon, x)=h(\varepsilon, x),
$$

where $\tilde{\mathcal{L}}_{\varepsilon}$ is defined by (6). In [4], [8], equations of the form

$$
\Delta_{\varepsilon} y(\varepsilon, x)=h(x)
$$




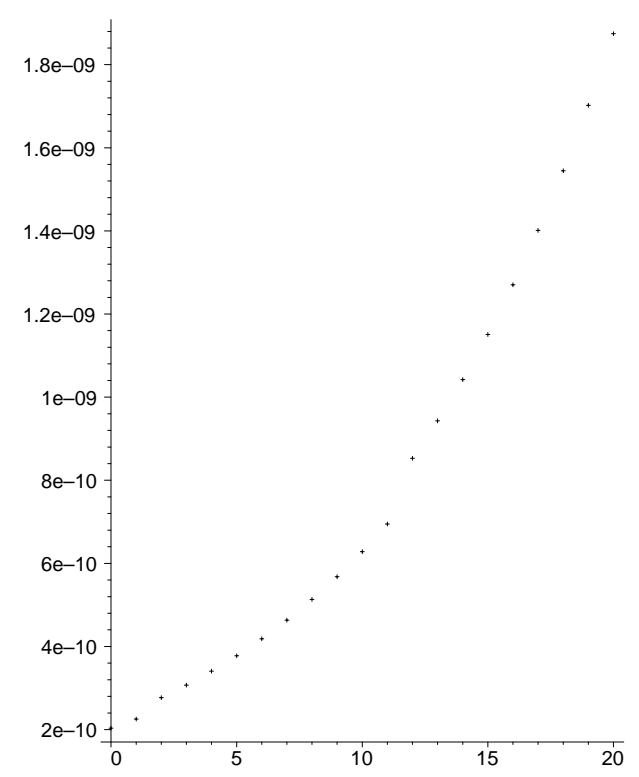

Figure 2: The function $a_{i}-a_{(i+10)}$

where $\Delta_{\varepsilon}$ is given by

$$
\Delta_{\varepsilon} y=\frac{y(x+\varepsilon)-y(x)}{\varepsilon},
$$

were treated. For the convenience of the reader, we recall this discussion and some definitions.

Definition 3 [8] (a) A complex domain $D$ is called horizontally convex if for all $x, x^{\prime}$ in $D$ with same imaginary part the line $\left[x, x^{\prime}\right]$ is a subset of $D$.

(b) A path $\gamma: I \subset \mathbb{R} \rightarrow \mathbb{C}$ is called $c$-ascending, $c>0$ if

$$
\forall t_{1}, t_{2} \in I \quad\left(t_{1}<t_{2} \Rightarrow \operatorname{Im}\left(\gamma\left(t_{2}\right)-\gamma\left(t_{1}\right)\right) \geq c\left|\gamma\left(t_{2}\right)-\gamma\left(t_{1}\right)\right|\right) .
$$

(c) A simply connected domain $\Omega$ is called c-ascending if there are unique $x^{+}$and $x^{-}$in $\mathrm{Cl}(\Omega)$ ( maybe infinite) that are of respective maximal and minimal imaginary parts in $\Omega$ and if the boundary of $\Omega$ consists of two c-ascending paths from $x^{-}$to $x^{+}$. The points $x^{+}$ and $x^{-}$are called the extreme points of $\Omega$.

Here $\operatorname{Cl}(\Omega)$ denotes the closure of $\Omega$.

Theorem 4 [8] Suppose that $\Omega \subset \mathbb{C}$ is a bounded simply connected domain that is $c$ ascending with $c \leq 1 / 2$. We call its extreme points $x^{ \pm}$. We fix some $\left.\left.\varepsilon \in\right] 0,1\right]$ and put

$$
\Omega_{\varepsilon}=\{x+\varepsilon s \mid x \in \Omega, s \in[-1 / 2,1 / 2]\}=\Omega+[-\varepsilon / 2, \varepsilon / 2] .
$$

Let $\overline{\mathcal{H}}\left(\Omega_{\varepsilon}\right)$ be the space of the holomorphic and bounded functions $h: \Omega_{\varepsilon} \rightarrow \mathbb{C}$. Then there exists a bounded linear operator $V_{\varepsilon}$, on $\overline{\mathcal{H}}\left(\Omega_{\varepsilon}\right)$, that is a right inverse of $\Delta_{\varepsilon}$. Moreover, for some $t \in[-1 / 8,1 / 8]$

$$
\text { - } V_{\varepsilon} h(x)=4 \int_{-1 / 8}^{1 / 8} \tilde{V}_{t} h(x) d t \text {, }
$$


- $\tilde{V}_{t} h(x)=\tilde{S}_{t} h(x)-\varepsilon \tilde{U}_{t} h(x)$,

- $\tilde{S}_{t} h(x)=\int_{\gamma_{x, t}^{-}} h(\xi) d \xi$,

- $\tilde{U}_{t} h(x)=\frac{1}{\varepsilon} \int_{\gamma_{x, t}^{-}} \frac{h(\xi)}{1-e_{x}(\varepsilon, \xi)} d \xi-\frac{1}{\varepsilon} \int_{\gamma_{x, t}^{+}} \frac{h(\xi)}{1-1 / e_{x}(\varepsilon, \xi)} d \xi$

- $\gamma_{x, t}^{-}$is an ascending path joining $x^{-}+\varepsilon t$ and $x-\varepsilon / 2$ (avoiding $x-\varepsilon$ and $x$ ) and such that $\operatorname{Im} \xi$ is increasing as $\xi$ varies on it.

- $\gamma_{x, t}^{+}$is an ascending path joining $x-\varepsilon / 2$ and $x^{+}+\varepsilon t$ (avoiding $x-\varepsilon$ and $x$ ) and such that $\operatorname{Im} \xi$ is increasing as $\xi$ varies on it.

- the function $e_{x}$ is given by $e_{x}(\varepsilon, \xi)=\exp \left(\frac{2 i \pi}{\varepsilon}(\xi-x)\right)$.

We refer to [8] for a detailed discussion of the paths.

Consider now the operator $\tilde{\mathcal{L}}_{\varepsilon}$ defined by $(6)$ and the equation $\tilde{\mathcal{L}}_{\varepsilon} f=h$. We reduce this equation to the previous one by "variation of constants". Suppose we know some function $Z(\varepsilon, x)$ satisfying $Z(\varepsilon, x+\varepsilon)=\frac{Z(\varepsilon, x)}{x+\varepsilon A(x)}$. Then $\tilde{\mathcal{L}}_{\varepsilon} f=h$ is equivalent to $\Delta_{\varepsilon} \tilde{f}=\tilde{h}$, $\tilde{f}(\varepsilon, x)=Z(\varepsilon, x) f(\varepsilon, x), \tilde{h}(\varepsilon, x)=\frac{Z(\varepsilon, x) h(\varepsilon, x)}{x+\varepsilon A(x)}$. Thus we obtain the following:

Given the same hypothesis as theorem 4 , but where $\Omega$ is in the right half-plane $\operatorname{Re} x>0$, there exists a linear operator $T_{\varepsilon}$, on $\overline{\mathcal{H}}\left(\Omega_{\varepsilon}\right)$, that defines a right inverse of $\tilde{\mathcal{L}}_{\varepsilon}$ and it is given by

$$
T_{\varepsilon} h(\varepsilon, x)=\frac{1}{Z(\varepsilon, x)} V_{\varepsilon}\left(\frac{Z(\varepsilon, x) h(\varepsilon, x)}{x+\varepsilon A(x)}\right)
$$

where $Z(\varepsilon, x)$ is as above and to be discussed below.

\subsection{Discussion of Boundedness of $T_{\varepsilon}$}

In general, $T_{\varepsilon}$ will not be uniformly bounded with respect to $\varepsilon$ as was the case for $V_{\varepsilon}$. However, we could modify it somewhat to obtain new right inverse operators $T_{\varepsilon}^{ \pm}$of $\tilde{\mathcal{L}}_{\varepsilon}$ that are uniformly bounded on $\bar{H}\left(D_{\varepsilon}^{ \pm}\right), D_{\varepsilon}^{ \pm}$being some subdomains of $\Omega_{\varepsilon}$ to be discussed in the next section. To do this, we have to take into consideration the new function $Z(\varepsilon, x)$ that enters into the integrals defining $T_{\varepsilon}$. If we let

$$
u(\varepsilon, x)=\varepsilon \log Z(\varepsilon, x),
$$

then $u$ satisfies

$$
\Delta_{\varepsilon} u(\varepsilon, x)=-\log (x+\varepsilon A(x)) .
$$

Whenever such a function $u$ exists and is uniformly bounded with respect to $\varepsilon$ on some compact $x$-domain, we say that it is a sum [4] of the complex analytic function $-\log (x+$ $\varepsilon A(x))$ on this $x$-domain. Moreover, it is known that a sum, when it exists, of a certain complex analytic function is close to an antiderivative of that function, with a difference of the order of $\varepsilon$. Thus, a sum of $-\log (x+\varepsilon A(x))$ satisfies

$$
u(\varepsilon, x)=x-x \log (x)-1+\mathcal{O}(\varepsilon),
$$


on some compact $x$-domain containing $x=1$.

Using (19), $Z(\varepsilon, x)$ satisfies

$$
Z(\varepsilon, x)=\exp \left(\frac{1}{\varepsilon}(x-x \log x-1)+\mathcal{O}(1)\right) .
$$

This implies

$$
\frac{Z(\varepsilon, \xi)}{Z(\varepsilon, x)}=\mathcal{O}\left(\exp \left(\frac{1}{\varepsilon}\left(R_{0}(x)-R_{0}(\xi)\right)+\mathcal{O}(1)\right)\right)
$$

where $R_{0}$ is the corresponding relief function, i.e. the surface described by

$$
\mathbb{R}^{2} \rightarrow \mathbb{R},(a, b) \longmapsto R_{0}(a+i b),
$$

where

$$
R_{0}(x)=\operatorname{Re}(x \log x-x+1) .
$$

Considering the relief of $R_{0}(x)$ in the right half-plane (see figure 3 below), we see that the

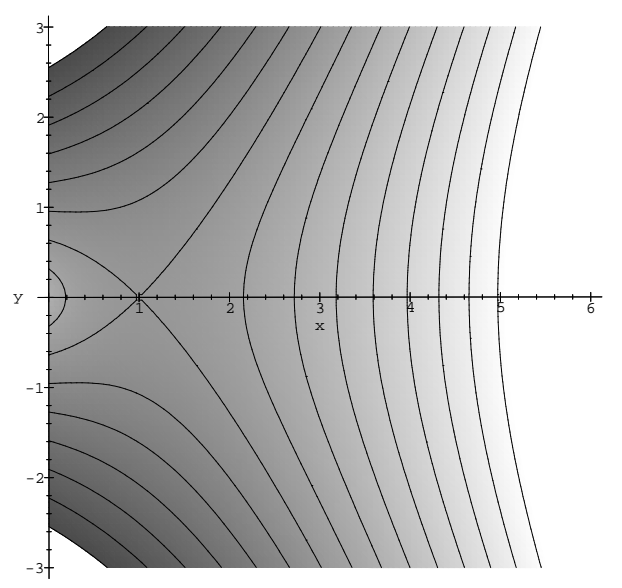

Figure 3: Real part of $x \log x-x+1$

level curves passing through the point $x=1$ divide the right half-plane into four regions, two mountains and two valleys. The mountains are to the left and the right of $x=1$. We denote the mountain situated to the left of $x=1$ by $M_{L}$ and the one to its right by $M_{R}$. We also call $V_{N}$ the valley situated to the north of $x=1$ and $V_{S}$ the one to its south. As already mentionned, we want to construct a domain $D$ that is horizontally convex and $c$-ascending with extreme points $x^{-}$and $x^{+}$of minimal and maximal imaginary parts respectively. It is easy to see that there exist some points $x_{i}$ for which there is no path, joining $x^{-}$to $x_{i}$, along which $R_{0}(\xi)$ decreases; that is why $T_{\varepsilon}$ is not anymore bounded on $\overline{\mathcal{H}}\left(\Omega_{\varepsilon}\right)$. However, we notice that the level curves of $R_{0}(x)$ always decrease whenever we go up from the level curve that is close to zero to the level curve at $1-\delta, \delta$ some positive small number. This is also the case whenever we go down from the $\infty$-level to that passing through $1+\delta$. It is then natural to set $x_{0}^{-} \in \mathbb{R}$ to be close to zero but less than one and $x_{0}^{+} \in \mathbb{R}$ to be greater than 1 but finite and to replace the integration path of the first integral defining $T_{\varepsilon}$ by some $c$-ascending path going either from $x_{0}^{-}$to $x-\varepsilon / 2$ or from $x_{0}^{+}$ to $x-\varepsilon / 2$. Then

$$
\int_{x_{0}^{-}}^{x-\varepsilon / 2} \frac{Z(\varepsilon, \xi) h(\varepsilon, \xi)}{Z(\varepsilon, x)(\xi+\varepsilon A(\xi))} d \xi
$$


is bounded except for those $x$ on $M_{R}$.

Similarly,

$$
\int_{x_{0}^{+}}^{x-\varepsilon / 2} \frac{Z(\varepsilon, \xi) h(\varepsilon, \xi)}{Z(\varepsilon, x)(\xi+\varepsilon A(\xi))} d \xi
$$

is bounded except for those $x$ on $M_{L}$.

We consider

$$
\int_{x^{-}+\varepsilon t}^{x-\varepsilon / 2} \frac{Z(\varepsilon, \xi) h(\varepsilon, \xi)}{Z(\varepsilon, x)(\xi+\varepsilon A(\xi))\left(1-e_{x}(\varepsilon, \xi)\right)} d \xi
$$

For $x$ sufficiently far away from $x^{-}$, and by (22) and (23), we obtain

$$
\frac{Z(\varepsilon, \xi)}{Z(\varepsilon, x)\left(1-e_{x}(\varepsilon, \xi)\right)}=\mathcal{O}\left(\exp \left(\frac{1}{\varepsilon}\left(R_{1}(x)-R_{1}(\xi)\right)\right)\right)
$$

where

$$
R_{1}(x)=\operatorname{Re}(x \log x-x+1+2 i \pi x) .
$$

As the relief $R_{1}(x)$ shows (see figure 4 below), this integral is not bounded unless $x^{-}$is

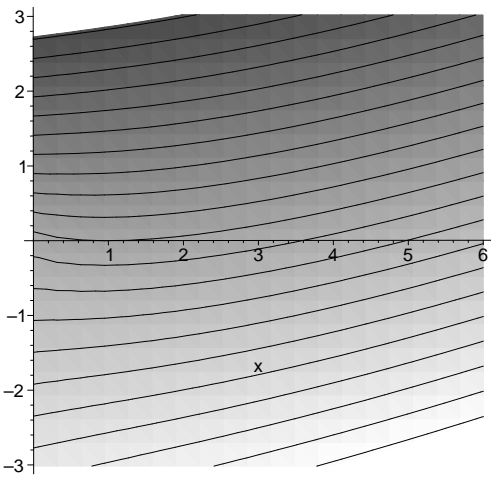

Figure 4: Real part of $x \log x-x+1+2 i \pi x$

chosen to lie on or above the level curve of $R_{1}$, that passes through $x_{0}^{+}$. Similarly, for $x$ sufficiently away from $x^{+}$,

$$
\frac{Z(\varepsilon, \xi)}{Z(\varepsilon, x)\left(1-e_{x}^{-1}(\varepsilon, \xi)\right)}=\mathcal{O}\left(\exp \left(\frac{1}{\varepsilon}\left(R_{2}(x)-R_{2}(\xi)\right)\right)\right)
$$

where

$$
R_{2}(x)=\operatorname{Re}(x \log x-x+1-2 i \pi x) .
$$

Consequently,

$$
\int_{x-\varepsilon / 2}^{x^{+}+\varepsilon t} \frac{Z(\varepsilon, \xi) h(\varepsilon, \xi)}{Z(\varepsilon, x)(\xi+\varepsilon A(\xi))\left(1-e_{x}^{-1}(\varepsilon, \xi)\right)} d \xi,
$$

could be bounded if $x^{+}$is chosen to lie on or below the level curve of $R_{2}$ (see figure 5 on page 12) that passes through $x_{0}^{+}$. Recall that $x^{-}$is of minimal imaginary part and $x^{+}$of maximal imaginary part. 


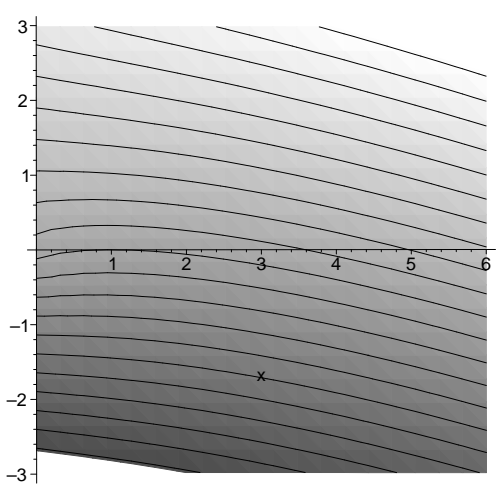

Figure 5: Real part of $x \log x-x+1-2 i \pi x$

\subsection{Description of $D, D^{-}$and $D^{+}$}

For reasons to be understood later, if we want to construct a bounded right inverse of $\mathcal{L}_{\varepsilon}$, we still need to impose further conditions on $x^{-}$and $x^{+}$. We need to choose $x^{-}$in $V_{S}$ and $x^{+}$in $V_{N}$. We also choose

- $\left.y_{N}^{-} \in\right] x^{+}, x_{0}^{+}\left[\cap V_{N}\right.$ such that the segment $\left[1-\delta, y_{N}^{-}\right]$is $c$-ascending.

- $\left.y_{S}^{-} \in\right] x^{-}, x_{0}^{+}\left[\cap V_{S}\right.$ such that the segment $\left[y_{S}^{-}, 1-\delta\right]$ is $c$-ascending.

- $\left.y_{N}^{+} \in\right] x_{0}^{-}, x^{+}\left[\cap V_{S}\right.$ such that the segment $\left[1+\delta, y_{N}^{+}\right]$is $c$-ascending.

- $\left.y_{S}^{+} \in\right] x_{0}^{-}, x^{-}\left[\cap V_{S}\right.$ such that the segment $\left[y_{S}^{+}, 1+\delta\right]$ is $c$-ascending.

Then we define

- $D$ to be the region enclosed within the four segments $\left[x_{0}^{-}, x^{+}\right],\left[x^{+}, x_{0}^{+}\right],\left[x_{0}^{+}, x^{-}\right]$, and $\left[x^{-}, x_{0}^{-}\right]$.(See figure 6 on page 13 )

- $D^{-}$to be the region enclosed within the six segments $\left[x_{0}^{-}, x^{+}\right],\left[x^{+}, y_{N}^{-}\right],\left[y_{N}^{-}, 1-\delta\right]$, and $\left[1-\delta, y_{S}^{-}\right],\left[y_{S}^{-}, x^{-}\right]$and $\left[x^{-}, x_{0}^{-}\right] .($See figure 7 on page 14$)$

- $D^{+}$to be the region enclosed within the six segments $\left[x_{0}^{+}, x^{+}\right],\left[x^{+}, y_{N}^{+}\right],\left[y_{N}^{+}, 1+\delta\right]$, $\left[1+\delta, y_{S}^{+}\right],\left[y_{S}^{+}, x^{-}\right]$and $\left[x^{-}, x_{0}^{+}\right]$. (See figure 8 on page 15 )

So, our domains $D, D^{-}$and $D^{+}$are all bounded horizontally convex and $c$-ascending.

\subsection{Bounded Right Inverse of $\tilde{\mathcal{L}}_{\varepsilon}$}

Keeping the same terminology as before, we put $D_{\varepsilon}=D+[-\varepsilon / 2, \varepsilon / 2]$ and $D_{\varepsilon}^{ \pm}=D^{ \pm}+$ $[-\varepsilon / 2, \varepsilon / 2]$ respectively. For $x \in D_{\varepsilon}$ and some $t \in[-1 / 8,1 / 8]$, we define $T_{\varepsilon}^{-}$and $T_{\varepsilon}^{+}$by

$T_{\varepsilon}^{ \pm} h(\varepsilon, x)=\int_{\gamma_{\gamma_{0}^{ \pm}}^{ \pm}} \frac{Z(\varepsilon, \xi) h(\varepsilon, \xi)}{Z(\varepsilon, x)(\xi+\varepsilon A(\xi))} d \xi-4 \int_{-1 / 8}^{1 / 8} \int_{\tilde{\gamma}_{x, t}^{-}} \frac{Z(\varepsilon, \xi) h(\varepsilon, \xi)}{Z(\varepsilon, x)(\xi+\varepsilon A(\xi))\left(1-e_{x}(\varepsilon, \xi)\right)} d \xi d t$ 


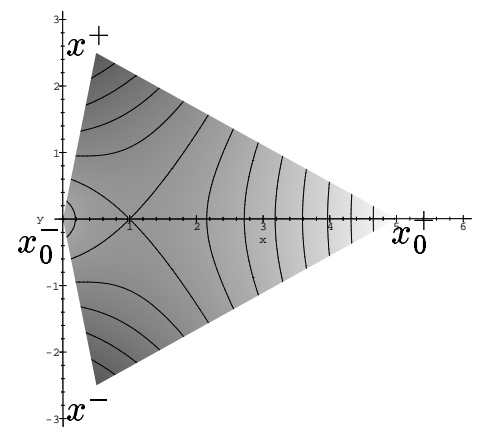

Figure 6: The domain $D$

$$
+4 \int_{-1 / 8}^{1 / 8} \int_{\tilde{\gamma}_{x, t}^{+}} \frac{Z(\varepsilon, \xi) h(\varepsilon, \xi)}{Z(\varepsilon, x)(\xi+\varepsilon A(\xi))\left(1-e_{x}^{-1}(\varepsilon, \xi)\right)} d \xi d t
$$

but where

- $\gamma_{x_{0}^{ \pm}}^{ \pm}$are some integration paths from $x_{0}^{ \pm}$to $x-\varepsilon / 2$ respectively.

- $\tilde{\gamma}_{x, t}^{-}$is some $c$-ascending path joining $x^{-}+\varepsilon t$ to $x-\varepsilon / 2$ (avoiding $x-k \varepsilon, k \in \mathbb{Z}$ ) and along which the function $R_{1}$ is decreasing.

- $\tilde{\gamma}_{x, t}^{+}$is some $c$-ascending path joining $x-\varepsilon / 2$ to $x^{+}+\varepsilon t$ (avoiding $x-k \varepsilon, k \in \mathbb{Z}$ ) and along which the function $R_{2}$ is increasing.

We show in the result below the existence of such paths and the boundedness of $T_{\varepsilon}^{ \pm}$on $\overline{\mathcal{H}}\left(\Omega_{\varepsilon}^{ \pm}\right)$respectively.

Theorem 5 The linear operators $T_{\varepsilon}^{ \pm}$define right inverse operators of $\tilde{\mathcal{L}}_{\varepsilon}$ on $\overline{\mathcal{H}}\left(\Omega_{\varepsilon}^{ \pm}\right)$. Moreover, for $x \in D_{\varepsilon}^{ \pm}$, there exist such above paths $\gamma_{x_{0}^{ \pm}}^{ \pm}$along which $R_{0}$ is decreasing and so making $T_{\varepsilon}^{ \pm}$bounded on $\overline{\mathcal{H}}\left(\Omega_{\varepsilon}^{ \pm}\right)$respectively.

Proof. The two operators $T_{\varepsilon}^{ \pm}$are also right inverses of $\tilde{\mathcal{L}}_{\varepsilon}$ since they differ from $T_{\varepsilon}$ by just a constant times a homogeneous solution of $\tilde{\mathcal{L}}_{\varepsilon} h(\varepsilon, x)=0$ and which is $1 / Z(\varepsilon, x)$. To show that $T_{\varepsilon}^{ \pm} h(\varepsilon, x)$ is bounded on $\overline{\mathcal{H}}\left(\Omega_{\varepsilon}^{ \pm}\right)$respectively, we may, without loss of generality, assume that $x-\varepsilon / 2 \in D_{\varepsilon}$ for otherwise $(x+\varepsilon)-\varepsilon / 2=x+\varepsilon / 2 \in D_{\varepsilon}$ and we show instead that $T_{\varepsilon}^{ \pm} h(\varepsilon, x+\varepsilon)$ is indeed bounded and so would be $T_{\varepsilon}^{ \pm} h(\varepsilon, x)$ since $T_{\varepsilon}^{ \pm} h(\varepsilon, x)=$ $\left(T_{\varepsilon}^{ \pm} h(\varepsilon, x+\varepsilon)-\varepsilon h(\varepsilon, x)\right) /(x+\varepsilon A(x))$ respectively. We write $x-\varepsilon / 2=\bar{x}+\varepsilon \bar{\alpha}$ where $\bar{x} \in D$ and $\bar{\alpha} \in[-1 / 2,1 / 2]$. Then since $D$ is a $c$-ascending domain, there exist some $c$-ascending paths $\gamma_{\bar{x}}^{-}$joining $x^{-}$to $\bar{x}$ and $\gamma_{\bar{x}}^{+}$joining $\bar{x}$ to $x^{+}$. Moreover, as the relief functions $R_{1}$ and $R_{2}$ show, $\gamma_{\bar{x}}^{-}\left(\gamma_{\bar{x}}^{+}\right)$could be chosen such that $R_{1}(\xi)$ decreases $\left(R_{2}(\xi)\right.$ increases $)$ as $\xi$ varies on it. 


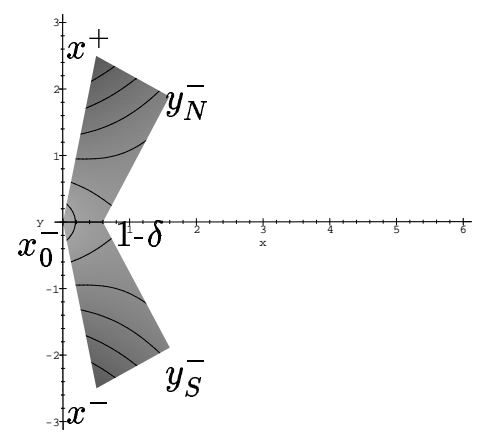

Figure 7: The domain $D^{-}$

The paths $\tilde{\gamma}_{x, t}^{ \pm}$are chosen in two ways according to whether $x$ is near $x^{-}$or $x^{+}$, or far away from them.

In case $x$ is not near $x^{-}$nor $x^{+}, \tilde{\gamma}_{x, t}^{ \pm}$could be chosen close to $\gamma_{\bar{x}}^{ \pm}$respectively with a distance of the order of $\varepsilon$. This implies then, on the basis of earlier discussion, that the second and the third integrals in the definition of $T_{\varepsilon}^{ \pm}$become bounded on $\overline{\mathcal{H}}\left(\mathcal{D}_{\varepsilon}^{ \pm}\right)$respectively.

In the case where $x$ is either near $x^{-}$or $x^{+}$, this technique no longer works. Some polygonal paths must be considered. For example, suppose that $x$ is near $x^{-}$, say that $\operatorname{Im} x \in$ $\left[\operatorname{Im} x^{-}, \operatorname{Im} x^{-}+\varepsilon c / 8\right]$, then

$$
\int_{-1 / 8}^{1 / 8} \int_{\tilde{\gamma}_{x, t}^{+}} \frac{Z(\varepsilon, \xi) h(\varepsilon, \xi)}{Z(\varepsilon, x)(\xi+\varepsilon A(\xi))\left(1-e_{x}^{-1}(\varepsilon, \xi)\right)} d \xi d t
$$

remains to be bounded. Then $\tilde{\gamma}_{x, t}^{-}$could be exactly chosen as in [8], [9] so that

$$
\int_{-1 / 8}^{1 / 8} \int_{\tilde{\gamma}_{x, t}^{-}} \frac{Z(\varepsilon, \xi) h(\varepsilon, \xi)}{Z(\varepsilon, x)(\xi+\varepsilon A(\xi))\left(1-e_{x}(\varepsilon, \xi)\right)} d \xi d t,
$$

is also bounded.

So far, we haven't yet shown, what makes our operators $T_{\varepsilon}^{ \pm}$more special than $T_{\varepsilon}$. Considering the first integral, the integration path $\gamma_{x_{0}}^{ \pm}$could be chosen as follows. As before, we put $x=\bar{x}+\varepsilon \bar{\alpha}$. Then if $x \in D_{\varepsilon}^{-}$, there exists $\gamma_{\overline{x_{0}}}^{-}$joining $x_{0}^{-}$to $x$ and lying in $D^{-}$such that the relief function $R_{0}$ is decreasing along it. This could be easily explained by the behavior of $R_{0}$. Then $\gamma_{x_{0}}^{-}$could be chosen close to $\gamma_{\bar{x}_{0}}^{-}$with a difference of the order of $\varepsilon$. Similarly, if $x \in D_{\varepsilon}^{+}$, there exists $\gamma_{\bar{x}_{0}}^{+}$joining $x_{0}^{+}$to $x$ and lying in $D^{+}$and such that $R_{0}(\xi)$ decreases as $\xi$ varies from $x_{0}^{+}$to $x$. Then $\gamma_{x_{0}}^{+}$is also chosen close enough to $\gamma_{\bar{x}_{0}}^{+}$. Then since

$$
\frac{Z(\varepsilon, \xi)}{Z(\varepsilon, x)}=\mathcal{O}\left(\exp \left(\frac{1}{\varepsilon}\left(R_{0}(x)-R_{0}(\xi)\right)+O(1)\right)\right)
$$




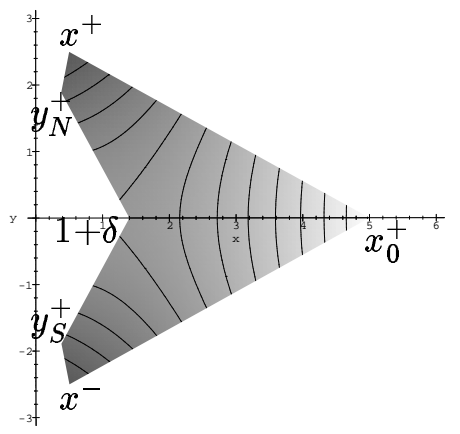

Figure 8: The domain $D^{+}$

the integral

$$
\int_{\gamma_{x_{0}}^{ \pm}} \frac{Z(\varepsilon, \xi) h(\varepsilon, \xi)}{Z(\varepsilon, x)(\xi+\varepsilon A(\xi))} d \xi
$$

becomes bounded on $\overline{\mathcal{H}}\left(\mathcal{D}_{\varepsilon}^{ \pm}\right)$respectively. This completes the proof.

Corollary 6 Given that $h(\varepsilon, x) \in \overline{\mathcal{H}}\left(\mathcal{D}_{\varepsilon}\right)$, equation(16) admits, on $D$, two analytic solutions $y^{ \pm}(\varepsilon, x)=T_{\varepsilon}^{ \pm} h(\varepsilon, x)$ that are bounded on $D^{ \pm}$respectively.

Proof. It is a direct application of theorem 5 .

\subsection{A Right Inverse of $\mathcal{L}_{\varepsilon}$}

Replacing $h(\varepsilon, x)$, in the linear "canard" equation (16), by $B(x) a(\varepsilon)+g(\varepsilon, x)$, we obtain

$$
y(\varepsilon, x+\varepsilon)=(x+\varepsilon A(x)) y(\varepsilon, x)+\varepsilon B(x) a(\varepsilon)+\varepsilon g(\varepsilon, x),
$$

where $A, B: D \longrightarrow \mathbb{C}$ are holomorphic and bounded, $\left.g:] 0, \varepsilon_{0}\right] \times D \longrightarrow \mathbb{C}$ is also holomorphic and bounded with $B(1) \neq 0$. Here $D$ is as described in section(3.2). This equation has, on $D$, two analytic solutions $y^{ \pm}(\varepsilon, x)=T_{\varepsilon}^{ \pm}(B(x) a(\varepsilon)+g(\varepsilon, x))$ that are bounded on $D^{ \pm}$respectively. We want to find $a(\varepsilon)$ for which equation $(26)$ has an analytic bounded solution on $D$. So, we solve for $a(\varepsilon)$,

$$
T_{\varepsilon}^{-}(B(x) a(\varepsilon)+g(\varepsilon, x))=T_{\varepsilon}^{+}(B(x) a(\varepsilon)+g(\varepsilon, x)) .
$$

We obtain

$$
a(\varepsilon)=-\int_{x_{0}^{-}}^{x_{0}^{+}} \frac{Z(\varepsilon, \xi) g(\varepsilon, \xi) d \xi}{\xi+\varepsilon A(\xi)} / \int_{x_{0}^{-}}^{x_{0}^{+}} \frac{Z(\varepsilon, \xi) B(\xi) d \xi}{\xi+\varepsilon A(\xi)}
$$


We define two new operators $T_{1}$ and $T_{2}$ on $\overline{\mathcal{H}}\left(\mathcal{D}_{\varepsilon}\right)$ by $T_{1}(g)=a$ and $T_{2}(g)=T_{\varepsilon}^{-}(B a+g)=$ $T_{\varepsilon}^{+}(B a+g)$, where $a$ is given by (27). Then we have the following result:

Theorem 7 The operator $\gamma_{\varepsilon}$, given by $\gamma_{\varepsilon} g(\varepsilon, x)=\left(T_{1} g, T_{2} g\right)$ defines a linear bounded right inverse operator of $\mathcal{L}_{\varepsilon}$ on $\overline{\mathcal{H}}\left(\mathcal{D}_{\varepsilon}\right)$

Proof. Note that the numerator of the right hand side of (27) is bounded above by $\left((2 \pi \varepsilon)^{1 / 2}\|g\|\left(x_{0}^{+}-x_{0}^{-}\right)\right) /(\min |\xi+\varepsilon A(\xi)|)$ and that its denominator behaves as $(2 \pi \varepsilon)^{1 / 2} B(1)$ as $\varepsilon \rightarrow 0$. Then there exists some constant $k$ such that $\left.|a(\varepsilon)| \leq k\|g\|, \forall \varepsilon \in] 0, \varepsilon_{0}\right]$. So, $T_{1}$ is bounded on $\overline{\mathcal{H}}\left(\mathcal{D}_{\varepsilon}\right)$. Recall that the two operators $T_{\varepsilon}^{-}$and $T_{\varepsilon}^{+}$, both defined on $\overline{\mathcal{H}}\left(\mathcal{D}_{\varepsilon}\right)$, are bounded on $\overline{\mathcal{H}}\left(\mathcal{D}_{\varepsilon}^{-}\right)$and $\overline{\mathcal{H}}\left(\mathcal{D}_{\varepsilon}^{+}\right)$respectively. Moreover, for this value for $a(\varepsilon)$, as given by (27), these two operators $T_{\varepsilon}^{-}$and $T_{\varepsilon}^{+}$act on $B(x) a(\varepsilon)+g(\varepsilon, x)$ in the same way. Then the commun value $T_{\varepsilon}^{-}(B(x) a(\varepsilon)+g(\varepsilon, x))=T_{\varepsilon}^{+}(B(x) a(\varepsilon)+g(\varepsilon, x))$ is an analytic solution of (26) on the whole domain $D$ and it is bounded on $D^{-} \cup D^{+}$. Since $D^{-} \cap D^{+} \neq \phi$, there is an appropriate subdomain $\tilde{D}$ of $D$ containing $D \backslash\left(D^{-} \cup D^{+}\right)$. Applying the maximum modulus principle on $\tilde{D}$, we obtain that $T_{2} g$ is an analytic bounded solution of (26) on $D$. This completes the proof.

Corollary 8 Given the same hypotheses as above, equation(26) has an analytic bounded solution $(a(\varepsilon), y(\varepsilon, x))$ given by $a=T_{1} g, y=T_{2} g$.

Proof. This is a direct application of theorem 7 .

\section{Analytic Solutions of Non Linear Equations on Bounded Domains}

We first have to discuss approximations of solutions of (1) as we want our solution to behave nicely as $\varepsilon \rightarrow 0+$. Putting formally $\varepsilon=0$, we find that necessarily $y=\mathcal{O}(\varepsilon)$. Comparing the coefficients of $\varepsilon$, we find that $y_{1}(x)=\lim _{\varepsilon \rightarrow 0+} \frac{1}{\varepsilon} y(\varepsilon, x)$ and $a_{0}=\lim _{\varepsilon \rightarrow 0+} a(\varepsilon)$, if they exist, have to satisfy

$$
y_{1}(x)=x y_{1}(x)+f\left(0, x, a_{0}, 0\right) .
$$

Hence a necessary condition for the existence of a well behaved solution is $f\left(0,1, a_{0}, 0\right)=0$. We assume in the sequel that such a value of $a_{0}$ exists and has been chosen. Without loss in generality, we can assume that $a_{0}=0$. Then $y_{1}(x)=f(0, x, 0,0) /(1-x)$ is analytic also at $x=1$. Thus we will prove.

Theorem 9 Let $\varepsilon_{0}>0$ be given and $D$ be some bounded domain satisfying the conditions of sections 3.1, 3.2 such that $f$ is defined and analytic on a neighborhood of $\left[0, \varepsilon_{0}\right] \times$ $c \ell(D) \times\{(0,0)\}$. We assume that $f(0,1,0,0)=0$ and $\frac{\partial f}{\partial a}(0,1,0,0) \neq 0$. Then, for $\varepsilon_{1}>0$ sufficiently small, equation(1) admits a family $\left\{\left(a_{\varepsilon}, y_{\varepsilon}\right)\right\}_{\left.\varepsilon \in] 0, \varepsilon_{1}\right]}$ of solutions such that $y_{\varepsilon}$ are analytic on $D$ and satisfy $y_{\varepsilon}(x)=\mathcal{O}(\varepsilon)$ uniformly on $D$.

Proof. We can assume, without loss of generality, that $f(0, x, 0,0)=0$ for all $x$. This can always be achieved by putting $y=\varepsilon y_{1}(x)+\tilde{y}$ with $y_{1}(x)$ as defined above the theorem. Indeed, the equation for $\tilde{y}$ is now

$$
\tilde{y}(\varepsilon, x+\varepsilon)=x \tilde{y}(\varepsilon, x)+\varepsilon \tilde{f}(\varepsilon, x, a(\varepsilon), \tilde{y}(\varepsilon, x)),
$$


where $\tilde{f}(\varepsilon, x, a, y)=f\left(\varepsilon, x, a, \varepsilon y_{1}(x)+y\right)+x y_{1}(x)-y_{1}(x+\varepsilon)$, and therefore $\tilde{f}(0, x, 0,0)=0$ for all $x$ by the definition of $y_{1}$.

As already mentioned, we want to solve equation (1) using the fixed point theorem. So, we rewrite it as

$$
y(\varepsilon, x+\varepsilon)=(x+\varepsilon A(x)) y(\varepsilon, x)+\varepsilon B(x) a(\varepsilon)+\varepsilon h(\varepsilon, x, a(\varepsilon), y(\varepsilon, x)),
$$

where

- $A(x)=\frac{\partial f}{\partial y}(0, x, 0,0)$,

- $B(x)=\frac{\partial f}{\partial a}(0, x, 0,0)$,

- $h(\varepsilon, x, a(\varepsilon), y(\varepsilon, x))=f(\varepsilon, x, a(\varepsilon), y(\varepsilon, x))-A(x) y(\varepsilon, x)-B(x) a(\varepsilon)$.

Defining $\psi(a, y)(\varepsilon, x)=h(\varepsilon, x, a(\varepsilon), y(\varepsilon, x))$, equation(1) reads

$$
\mathcal{L}_{\varepsilon}(a, y)(\varepsilon, x)=\psi(a, y)(\varepsilon, x)
$$

where the left-hand side of the equation denotes the main linear part of equation(1) and the right-hand side contains the remaining terms.

In the preceding section, we constructed a right inverse $\gamma_{\varepsilon}$ of $\mathcal{L}_{\varepsilon}$ on $D$ under the condition that $B(1)=\frac{\partial f}{\partial a}(0,1,0,0) \neq 0$ which is satisfied here. Then it is sufficient to solve the fixed point equation $(a, y)=\gamma_{\varepsilon} \psi(a, y)$.

We construct below a closed bounded subset $\mathcal{B}$ of a certain Banach space $\mathcal{E}$ on which we show that $\gamma_{\varepsilon} \psi$ is a contraction. First, we define

- $\left.E=\{a:] 0, \varepsilon_{1}\right] \rightarrow \mathbb{R} \mid a$ is some bounded scalar function of $\left.\varepsilon\right\}$,

- $\left.F=\{y:] 0, \varepsilon_{1}\right] \times K \rightarrow \mathbb{C} \mid y$ is holomorphic and bounded $\}$, boths endowed with the usual norm.

- $\mathcal{E}=E \times F$,

- $\mathcal{B}=\{(a, y) \in \mathcal{E}|| a|\leq \rho| y \mid, \leq \rho\}$

where $\varepsilon_{1}, \rho$ are to be determined below.

We have

$\psi\left(a_{2}, y_{2}\right)-\psi\left(a_{1}, y_{1}\right)=\int_{0}^{1} \frac{\partial \psi}{\partial y}\left(a_{1}, y_{1}+s\left(y_{2}-y_{1}\right)\right)\left(y_{2}-y_{1}\right) d s+\int_{0}^{1} \frac{\partial \psi}{\partial a}\left(a_{1}+s\left(a_{2}-a_{1}\right), y_{2}\right)\left(a_{2}-a_{1}\right) d s$

where

$$
\left[\frac{\partial \psi}{\partial y}(a, y) h\right](\varepsilon, x)=\left(\frac{\partial f}{\partial y}(\varepsilon, x, a(\varepsilon), y(\varepsilon, x))-A(x)\right) h(\varepsilon, x)
$$

and

$$
\left[\frac{\partial \psi}{\partial a}(a, y) b\right](\varepsilon, x)=\left(\frac{\partial f}{\partial a}(\varepsilon, x, a(\varepsilon), y(\varepsilon, x))-B(x)\right) b(\varepsilon) .
$$

Thus, by the definition of $A$ and $B$, we obtain for $(a, y) \in \mathcal{B}$

$$
\frac{\partial \psi}{\partial y}(a, y)=\mathcal{O}(\max (|\varepsilon|,|a|,|y|)) \leq C(|\varepsilon|+|a|+|y|) \leq C\left(\varepsilon_{1}+2 \rho\right)
$$


and

$$
\frac{\partial \psi}{\partial a}(a, y)=\mathcal{O}(\max (|\varepsilon|,|a|,|y|)) \leq C(|\varepsilon|+|a|+|y|) \leq C\left(\varepsilon_{1}+2 \rho\right),
$$

where $C$ is some constant (independent of $\varepsilon_{1}, \rho$ ).

Now denote the norm $L=\left|\gamma_{\varepsilon}\right|$. Choosing $\rho$ and $\varepsilon_{1}$ such that $2 C\left(\varepsilon_{1}+2 \rho\right) \leq \frac{1}{2 L}$, i.e. $\rho \leq \frac{1-4 C L \varepsilon_{1}}{8 C L}$, we obtain

$$
\left|\psi\left(a_{2}, y_{2}\right)-\psi\left(a_{1}, y_{1}\right)\right| \leq \frac{1}{2 L} \max \left(\left|y_{2}-y_{1}\right|,\left|a_{2}-a_{1}\right|\right)
$$

for all $\left(a_{1}, y_{1}\right),\left(a_{2}, y_{2}\right) \in \mathcal{B}$. As $f(0, x, 0,0)=0$ for all $x$ and $D$ is bounded, we can also choose $\varepsilon_{1}$ such that $\psi(0,0)(\varepsilon, x)=f(\varepsilon, x, 0,0)$ has a $F$-norm smaller than $\rho /(2 L)$.

This implies that $\gamma_{\varepsilon} \psi: \mathcal{B} \rightarrow \mathcal{B}$ is a contraction with a contraction factor at most $1 / 2$. Thus the existence of a fixed point $(a, y)$ of $\gamma_{\varepsilon} \psi$ and hence of a solution of (1) under the conditions of the theorem is proved.

\section{$5 \quad$ Future Directions of Research}

We end this work by mentioning possible future work to be pursued in the context of my Ph.D. research.

- Extend the discussions on the discrete solutions; for example treat nonlinear equations and prove the conjecture that all values of $a(\varepsilon, \theta), 0 \leq \theta \leq \varepsilon$ lie in some interval of length $\mathcal{O}\left(\varepsilon^{k+1}\right)$ if $f$ is $C^{k}$.

- Show exponential closeness also in the complex case.

- Extend the result to infinite domains; here, as for example in [7], canard solutions might be unique.

- Prove local results on canards for difference equations that are analogous to those for differential equations in [2].

\section{References}

[1] E. Benoît, J.L. Callot, F. Diener, M. Diener, Chasse au canard, Collect. Math., 31, 1-3 (1981) 37-119.

[2] M. Canalis-Durand, J.-P. Ramis, R. Schäfke, Y. Sibuya, Gevrey solutions of singularly perturbed differential equations, J. Reine Angew. Math. 518 (2000), 95-129.

[3] A. Fruchard, Canards et râteaux, Ann. Inst. Fourrier, Grenoble 42, 1-2, (1992), 825855 .

[4] A. Fruchard, The sum of a function, Analysis 16, (1996), 65-88.

[5] A. Fruchard, Sur l'équation aux différences affine du premier ordre unidimensionnelle, Ann. Inst. Fourrier, Grenoble 46, 1, (1996), 139-181. 
[6] A. Fruchard, R. Schäfke, Exponentially small splitting of separatrices for difference equations with small step size, J. Dynam. Control Systems 2, no.2 (1996), 193-238.

[7] A. Fruchard, R. Schäfke, Exceptional complex solutions of the forced van der Pol equation, Funkcialaj Ekvacioj 42, (1999), 201-223.

[8] A. Fruchard, R. Schäfke, Analytic solutions of difference equations with small step size, J. of Difference Equations and Applications 7, (2001), 651-684.

[9] A. Fruchard, R. Schäfke, Retard à la bifurcation et equations aux différences, prepint IRMA 7, rue René Descartes 67084 Strasbourg Cedex, France (2001).

[10] S. N. Elaydi, An Introduction To Difference Equations, Springer(1991). 